\title{
Soft X-ray tomographic reconstruction of JET ILW plasmas with tungsten impurity and different spectral response of detectors
}

\author{
Jan Mlynar ${ }^{\mathrm{a}}$, Matej Tomes ${ }^{\mathrm{a}, \mathrm{b}}$, Martin Imrisek ${ }^{\mathrm{a}, \mathrm{b}}$, Barry Alper ${ }^{\mathrm{c}}$, Martin O’Mullane ${ }^{\mathrm{c}}$, \\ Tomas Odstrcil $^{\mathrm{d}}$, Thomas Puetterich ${ }^{\mathrm{d}}$ and JET EFDA contributors ${ }^{*}$ \\ JET-EFDA, Culham Science Centre, Abingdon, OX14 3DB, UK \\ ${ }^{a}$ Institute of Plasma Physics AS CR, Za Slovankou 3, CZ-182 00 Prague 8, Czech Republic \\ ${ }^{b}$ Charles University, FMP, V Holesovickach 2, CZ-180 00 Prague 8, Czech Republic \\ ${ }^{c}$ Culham Centre for Fusion Energy, Abingdon, OX14 3DB, UK \\ ${ }^{d}$ Max-Planck-Institut für Plasmaphysik, D-85748 Garching, Germany \\ * See the Appendix of F. Romanelli et al., Proceedings of the 24th IAEA \\ Fusion Energy Conference 2012, San Diego (USA)
}

The Joint European Torus (JET) presently operates with the ITER-like wall (ILW) including tungsten divertor. Analyses of the soft X-ray radiation (SXR) data present an important tool for studies of transport of tungsten into JET plasmas. SXR spatial distribution can be measured by horizontal $(\mathrm{H})$ and two vertical $(\mathrm{V}, \mathrm{T})$ pinhole cameras. Tomographic reconstruction of SXR emissivity from their data is challenging due to different spectral sensitivities of the cameras which can cause significant inconsistencies due to spectral properties of tungsten radiation. In this contribution an advanced correction based on evaluation of the sensitivity differences for individual lines of view of the SXR detectors is presented. The adjustments take into account atomic data of tungsten as well as the SXR intensity perturbation due to tungsten influx. First results are presented and discussed.

Keywords: tokamak, plasma diagnostics, tomography, soft X-ray, tungsten radiation

\section{Introduction}

Spatially resolved soft X-ray (SXR) measurements represent an important tool for monitoring impurity profiles in JET plasmas with the all-metal ITER-like first wall. Line-integrated profile measurements are provided by three pinhole cameras, see figure 1 : camera $\mathrm{V}$ at octant 2 and camera $\mathrm{T}$ at octant 7 (both with vertical view, 35 channels and $250 \mu \mathrm{m}$ Be filters), and the radiation protected camera S4 at octant 4 (horizontal view, 16 channels, $350 \mu \mathrm{m}$ Be filter, $\sim 15^{\circ}$ tilt from the poloidal plane). The diagnostic is capable of high temporal resolution (up to $200 \mathrm{kHz}$ ).

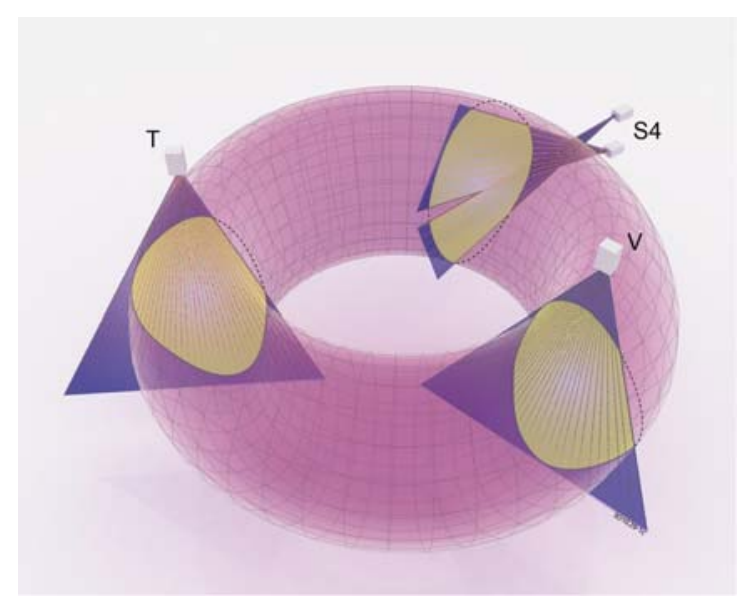

Fig. 1. Scheme of the layout of the SXR diagnostics at JET with respect to plasma geometry.
Tomographic inversion based on Minimum Fisher Regularisation method (MFR) [1] is applied on the line integrated data in order to derive two-dimensional spatial distribution of the SXR plasma emissivity in its poloidal cross-section. Although the SXR diagnostic setup at JET is not ideal for the tomographic reconstruction, significant and robust results have been obtained with MFR tomography, see e.g. [2], [3]. A possibility of correcting the MFR inputs for events with high temporal resolution due to different toroidal location of SXR cameras was presented in [4]. In this contribution, the MFR tomography is reviewed, a novel method for correcting the adverse effects of different spectral sensitivities of the cameras is proposed, and first results are presented. The studies have been motivated in particular by the observed data inconsistency between the SXR cameras during increased SXR radiation due to tungsten influx. In [5], this inconsistency was proved to stem from different spectral sensitivities of the JET SXR cameras. The corresponding systematic error, when not accounted for in the MFR, could cause major artifacts in the reconstruction or even prevent convergence of the algorithm.

\section{Implementing the correction coefficients}

\subsection{MFR tomography and its modification}

In plasma tomography relying on base functions the link between measured projections $f_{i}$ and unknown distribution of the local emissivities $g_{j}$ is established by a set of linear equations: 


$$
f_{i}=\sum_{j}^{N} T_{i j} g_{j}
$$

The contribution matrix $T_{i j}$ specifies weight of the $\mathrm{j}$-th source of radiation in the $i$-th line integrated measurement. In MFR, a smooth solution for the 2D plasma emissivity $g$ is found on a discrete rectangular mesh of pixels by minimising $\Lambda_{M F}=\chi^{2} / 2+\alpha I_{F}$ where $\chi^{2}$ is the goodness-of-fit derived via the Pearson's test using the expected data errorbars, $\alpha$ is a regularisation (smoothing) parameter and $I_{F}$ is the Fisher information of the reconstructed image: $I_{F}=\int(\nabla g)^{2} g^{-1} d S$. Anisotropic smoothness of the reconstruction with respect to the magnetic flux has been also introduced: $(\nabla g)^{2} \approx g \mathbf{H} g \quad$ where $\mathbf{H}=\mathbf{B}_{\|}^{T} e^{\eta} \mathbf{B}_{\|}+\mathbf{B}_{\perp}^{T} e^{-\eta} \mathbf{B}_{\perp}$. Here, $\mathbf{B}_{\|}$and $\mathbf{B}_{\perp}$ represent numerical differentiation matrices acting parallel and perpendicular to the magnetic flux, respectively. Notice that the anisotropy factor $\eta$ enforces preferential smoothness along the magnetic flux surfaces, allowing for steeper gradients in the reconstructed radial emissivity profile. For details, see e.g. [1].

Modification of the MFR to different spectral sensitivities of the cameras is quite straightforward provided that the spectral properties of the SXR plasma emission are known. Let us assume that MFR would be run on data from the vertical camera $\mathrm{V}$ and the horizontal camera S4 (for simplicity denoted by $\mathrm{H}$ in the following text). Their relative spectral sensitivities shall be fully determined by functions $S_{V}(v)$ and $S_{H}(v)$, respectively, where $v$ is the SXR radiation frequency. In the first approximation the difference between the two functions is exclusively due to the different $\mathrm{Be}$ foil thickness, see figure 2.

In general terms, the local plasma emissivity $g_{j}$ is dependent on the SXR frequency, too. Therefore, data from camera $\mathrm{V}$ correspond to

$$
f_{V i}=\int S_{V}(v) \sum_{j}^{N} T_{i j} g_{j}(v) d v=\sum_{j}^{N} T_{i j} \int S_{V}(v) g_{j}(v) d v
$$

Indeed, the contribution matrix $T_{i j}$ describes the geometric setup only, so that it is not a function of the SXR frequency. An analogous relation holds for the horizontal camera $\mathrm{H}$.

As it is impossible to observe $g_{j}(v)$ beyond the interval of the spectral sensitivity of either camera, the task of the tomography inversion should be limited to the spectral sensitivity of the least sensitive element, which is in our case the camera $\mathrm{H}$. Therefore, let us define the unknown distribution of SXR plasma emissivity in equation (1) by

$$
g_{j}=\int S_{H}(v) g_{j}(v) d v
$$

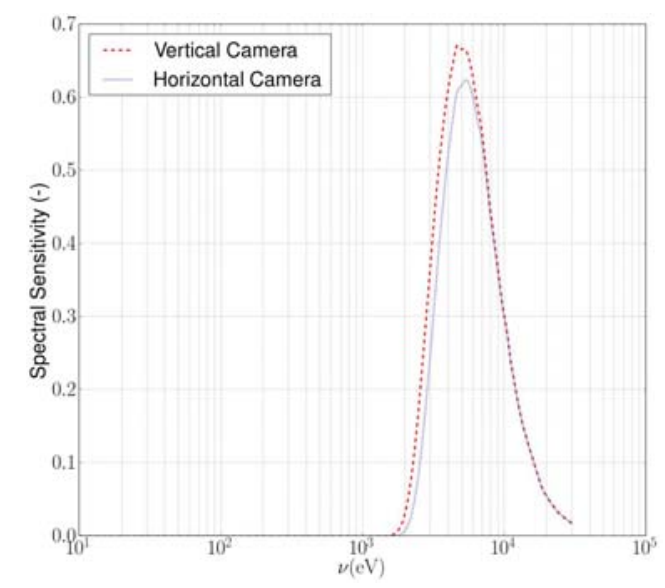

Fig. 2. Spectral sensitivities of the JET SXR camera V with $250 \mu \mathrm{m}$ Be filter, and of its camera $\mathrm{H}$ with $350 \mu \mathrm{m}$ Be filter

With this definition, equation (1) remains unchanged for the camera $\mathrm{H}$, while for the camera $\mathrm{V}$ the corresponding rows of the contribution matrix $T_{i j}$ have to be multiplied by the following correction factor:

$$
C_{j}=\frac{\int S_{H}(v) g_{j}(v) d v}{\int S_{V}(v) g_{j}(v) d v}
$$

Therefore, only the contribution matrix is modified, while equation (1) is valid so that the MFR tomography can be applied directly on data without any further amendments. However, besides knowledge of the functions $S_{H}(v)$ and $S_{V}(v)$ relation (4) also requires inputting the unknown characteristics of the local plasma SXR emissivity in its spectral dependence. The first possibility of resolving this complication relies in the forward-fitting procedures based on simplified plasma modeling, see e.g. [5]. As another option, in order to maintain the advantage of inverse reconstruction (in particular, little dependence on other diagnostic systems, on plasma models and on assumed plasma symmetries) it is proposed in this work to try and establish approximate values of the $C_{j}$ coefficients in an iterative procedure.

The method is reminiscent of the iterative minimization of the Fisher information in MFR [1]. In the first order estimate, all the $C_{j}$ coefficients are set equal to a single constant $C$ which is derived from the condition that the total power of the SXR radiation is camera independent. This estimate has been actually used as a standard for tomographic reconstructions in all the recent analyses, where it was observed that the constant $C$ can change its value considerably in time due to the tungsten influx. Based on this standard tomographic reconstruction, data from the ADAS atomic database and the plasma temperature profile the $C_{j}$ correction coefficients can be evaluated in better detail from (4). To this end, a method for estimating $g_{j}(v)$ from $g_{j}$ is outlined in the next section. A repeated run of tomographic reconstruction - with $C_{j}$ 's instead of a single $C$ value - would then provide a more dependable image of spatial distribution of the SXR radiation. 


\subsection{Spectral properties of the SXR radiation}

Since the ITER-like wall installation at JET, the SXR radiation in majority of plasma discharges prove to be dominated by bremsstrahlung which is occasionally overwhelmed by tungsten radiation [6]. With this knowledge, the simplest model of the sources of the SXR radiation can be written as follows:

$$
g_{j}(v)=D_{f f}+c_{W}\left(W_{f f}+W_{f b}+W_{L}\right)
$$

Here, $D_{f f}\left(v, T_{e}\right)$ is the deuterium plasma bremsstrahlung (in a more advanced model, this factor can include light impurities via $\left.Z_{e f f}\right)$, while $W_{f f}\left(v, T_{e}\right)$, $W_{f b}\left(v, T_{e}\right)$ and $W_{L}\left(v, T_{e}\right)$ correspond to free-free, freebound and line radiation of tungsten in a deuterium plasma with concentration $c_{W}=n_{w} / n_{e} \ll 1$. While the bremsstrahlung factors have been derived analytically, for the free-bound (recombination) and line radiation factors the OPEN-ADAS database has been used [7]. Furthermore, plasma temperature profile and tungsten concentration must be known in order to evaluate (5). While the former is uploaded from the JET database, the latter can be established from comparison of the atomic data model with the first-order estimate of the $g_{j}$ distribution. This method is illustrated on actual data analyses in part 3.

\section{Experimental results}

In the course of development and tests of the method as described in part 2, artificial as well as experimental data from JET were used [8]. In this contribution, three new and representative analyses of JET experimental data are presented which illustrate performance of the numerical correction of the systematic data error due to different spectral sensitivities of the JET SXR cameras.

First, results of the SXR radiation analyses in JET plasma discharge \#81835 are presented. In this discharge, negligible amount of heavy impurity was observed so that according to eq. (5) the separated influence of the bremsstrahlung factor $D_{f f}$ could have been tested, see figure 3. For simplicity, a constant plasma density has been assumed. Next, values of the correction factors $C_{j}$ are shown to illustrate their decrease with plasma temperature and their smooth and poloidally symmetrical distribution. In the tomography reconstruction, these corrections result in a horizontally thinner profile with a higher maximum, however, the amplitude of the correction is rather low, achieving several per cent of the peak emissivity.

Second, SXR radiation from the JET plasma discharge \#82722 during a major tungsten influx was analysed. The corresponding properites of SXR radiation in the initial phase of the influx (at $5.2 \mathrm{~s}$ ) are presented in figure 4 . In the left frame of this figure, the tungsten contribution to eq. (5) is shown instead of the $D_{f f}$, which behaves similarly to the previous example. It is clearly seen that at this moment the tungsten impurity is observed at the edge only, radiating with a significant horizontal asymmetry due to centrifugal forces in a toroidally rotating plasma [5]. Let us remind here that the presented tungsten radiation intensity is computed from (5) according to SXR radiation distribution provided by the standard tomographic reconstruction. Interestingly, the resulting correction coefficients achieve large values in the upper half of the plasma edge only. The subsequent corrected tomography reconstruction shows the peak emissivity radiating more towards the edge of the plasma. Besides, there is less SXR radiation attributed to the opposite side of the plasma edge, while the central radiation due to the hydrogen bremsstrahlung slightly increases like in the previous study of discharge \#81835. Again, the overall amplitude of the resulting correction is rather low, in the order of several per cent of the maximum emissivity.

Figure 5 shows analogous results for a later stage of the same JET discharge \#82722 at $7.5 \mathrm{~s}$, with tungsten reaching the plasma core resulting in the characteristic very peaked and intense radiation. Indeed, the distribution of tungsten radiation intensity (in the leftmost frame) is almost identical to the corrected tomography image in the third frame from the left. However, the resulting correction factors, which are influenced mainly by the tungsten line radiation in this case, prove to be almost constant across the plasma volume except the striking up-down asymmetry, see the second frame from the left in figure 5. Finally, the frame at right shows that the corrected tomography reconstruction features lower maximum level of the SXR
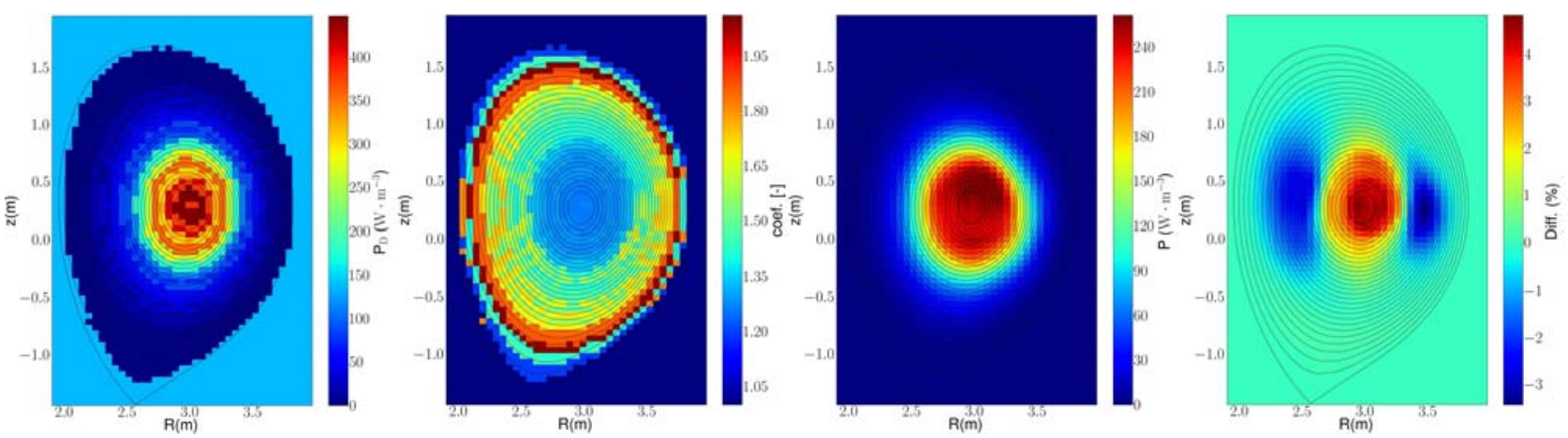

Fig. 3. Spatial characteristics of SXR radiation in JET discharge \#81835 at $11.1 \mathrm{~s}$, from L to R: bremsstrahlung intensity, correction coefficients $C_{j}$, corrected tomography reconstruction, difference between standard and corrected reconstruction. 

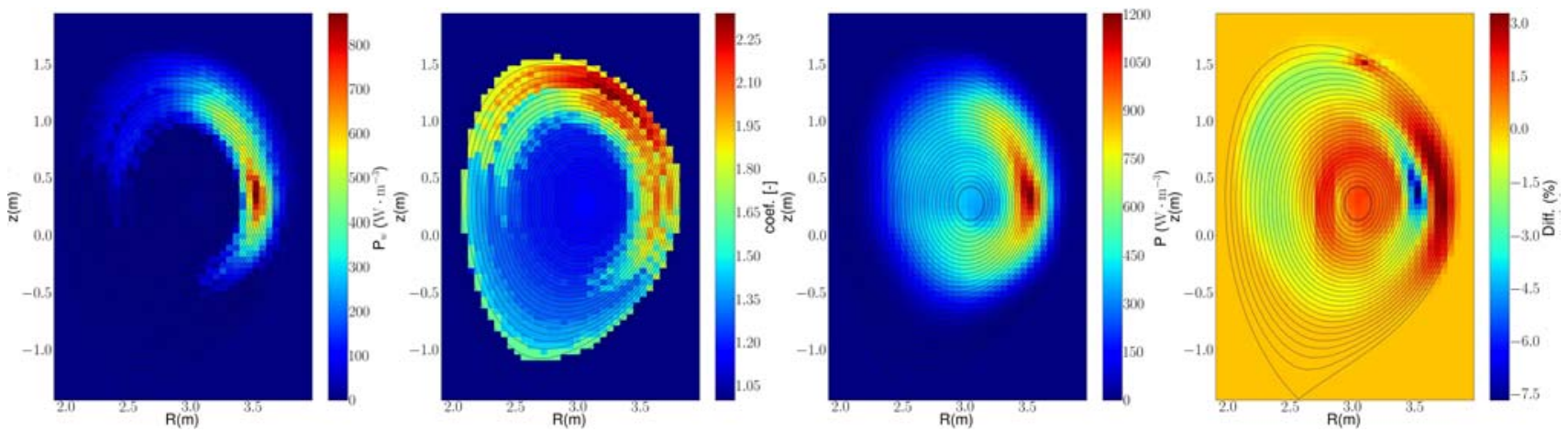

Fig. 4. Spatial characteristics of SXR radiation in JET discharge \#82722 at 5.2 s, from L to R: tungsten radiation intensity, correction coefficients $C_{j}$, corrected tomography reconstruction, difference between standard and corrected reconstruction.
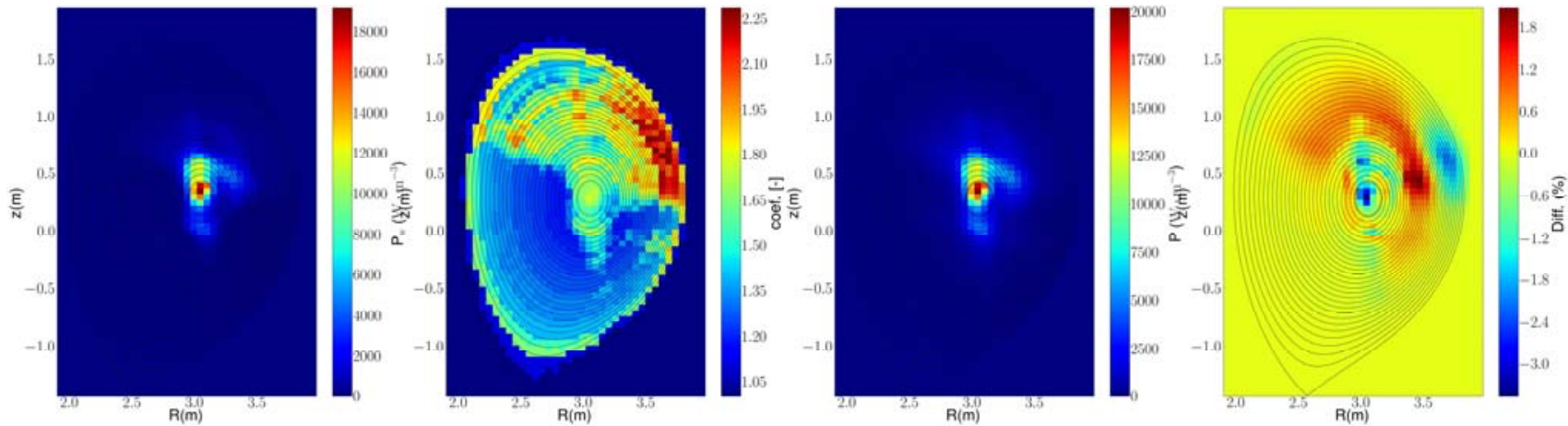

Fig. 5. Spatial characteristics of SXR radiation in JET discharge \#82722 at $7.5 \mathrm{~s}$, from L to R: tungsten radiation intensity, correction coefficients $C_{j}$, corrected tomography reconstruction, difference between standard and corrected reconstruction.

intensity and somewhat higher background. Even in this case, however, the relative effect of the correction is quite insignificant.

\section{Conclusions}

In this contribution, a novel method aimed at improving plasma tomography reconstruction by correcting different spectral sensitivities of SXR diagnostic cameras has been presented. Application of the method on experimental data demonstrated its robust and dependable performance, however, only with a rather small resulting correction with respect to the standard tomographic reconstruction, comparable to errors in experimental and atomic data. Importantly, this study demonstrated that even the computationally much lighter standard reconstruction (with no use of atomic data) results in minor systematic errors. It is to be mentioned here, that from 2015 JET SXR diagnostic system will be upgraded so that the $\mathrm{V}$ and $\mathrm{H}$ cameras have both $250 \mu \mathrm{m}$ Be foil filter. This shall fix the data consistency issue in the SXR tomography. Present study will however remain relevant for any previous data.

Interestingly, this study allows also for a rather basic quantitative estimate of the tungsten concentration according to (5). This feature has not been detailed in the contribution because we consider the forward-fitting procedure presented in [5] much better suited for this purpose, leading to similar results with higher precision. Nevertheless, it is worth mentioning that in the present work, a vertical asymmetry of the tungsten concentration is observed (see figures 4 and 5) while the forwardfitting procedure was constrained to a symmetry in this direction. The observed asymmetry has quite a low amplitude and therefore it might be an artifact due to the asymmetry of the SXR diagnostic setup. This result can be elucidated by an independent diagnostic system, e.g. by a spatially resolved tungsten spectrometry.

\section{Acknowledgments}

This work was supported by grant of the Czech Science Foundation GA P205/10/2055, by the MSMT project LG14002, by the MSMT project LM2011021 and EURATOM, and carried out within the framework of the European Fusion Development Agreement. The views and opinions expressed herein do not necessarily reflect those of the European Commission.

\section{References}

[1] M. Odstrcil et al., Nucl. Inst. Methods Phys. Res. A 686 (2012) 156

[2] C. Angioni et al., Nucl. Fus. 548 (2014) 083028

[3] P. Jacquet et al., Phys. Plasmas 216 (2014) 061510

[4] J. Mlynar et al., EPS Conf. Plasma Phys. ECA Vol 35G, P4.052/1 (2011)

[5] T. Pütterich et al., Proc. 24th IAEA FEC 2012, San Diego (USA), EX/P3-1

[6] T. Pütterich et al., Nucl Fus 50 (2010) 085016

[7] OPEN-ADAS, ADAS project, http://open.adas.ac.uk

[8] M. Tomes, MSc thesis, FEE, Czech Technical University in Prague, May 2014 